\title{
Combination of Biocontrol Agents for Controlling Soybean Damping-off Caused by Rhizoctonia solani Marwa A. M. Atwa
}

Legume and Forage Diseases Research Department. Plant Pathol. Res. Inst., Agric. Res. Centre, Giza, Egypt.

Goybean seeds (cv. Giza 22) were treated with Paenibacillus Ppolymyxa, Pseudomonas fluorescens and Mycorrhizeen compound (contains mainly endospores of Glomus sp.) as biocontrol agents either individually or in different combinations to study their effect on the infection with Rhizoctonia solani under greenhouse and field conditions. Treatment of Rizolex-T 50\% was also applied to compare the output data. Under greenhouse conditions, all the treatments significantly reduced the percentages of pre and postemergence damping-off and increased survived plants compared to untreated control grown in infested soil by $R$. solani. The highest percentage of survived plants $92 \%$ was achieved from the triple combination treatment (Mycorrhizeen plus $P$. polymyxa and $P$. fluorescens) and Rizolex-T treatment as compared to the untreated control, which achieved 48\%. The significant increases in plant height, shoot dry weight and root dry weight of soybean plants were recorded with the triple combination treatment followed by the fungicide treatment. Meantime, all treatments significantly enhancing both number of nodules/plant and total dry weight of nodules/plant compared with untreated control grown in infested soil by $R$. solani. The maximum increase was observed with triple combination treatment followed by the dual combination of (Mycorrhizeen plus $P$. fluorescens) then Mycorrhizeen. In addition, the results showed that combined treatments enhanced the percentage of colonization of mycorrhizae in the roots over Mycorrhizeen sole treatment as $18.7 \%$ for the triple combination treatment. Under field conditions at two locations, Giza and Etai El-Baroud Agric. Res. Stat. during the summer of 2017 growing season, the triple combination treatment resulted in high values of disease control near to that obtained with Rizolex- T treatment. Such an effect was true for pre and postemergence damping-off as well as the percentage of survived plants. Meantime, all treatments significantly showed stimulatory effect for crop parameters of soybean plants i.e. plant height, number of branches/plant, number of pods/plant, seed weight $(\mathrm{g}) /$ plant and seed yield ( $\mathrm{kg} /$ feddan) compared to the untreated control treatment in the two locations. The activity of oxidative enzymes and phenolic compounds contents were determined in soybean plants treated with 
the different bio-agents either individually or in different combinations. All treatments increased the peroxidase (PO), polyphenol oxidase (PPO) and phenolic content in the treated soybean plants over the untreated control plants sown in infested soil with $R$. solani. The maximum increase in the activity of both enzymes and phenolic contents was recorded with the triple combination treatment followed by the two dual treatments of the Mycorrhizeen.

Keywords: Crop parameters, Damping-off, Bioagents, enzymatic activity, Mycorrhizeen, phenolic contents.

Soybean (Glycine max L. Merril) is one of the most important pulse crops in the world. However, soybean is subjected to infection by Rhizoctonia solani Kühn which causes seedling blight (pre- and post-emergence damping-off) and root rot (Xue et al., 2007 and Chang et al., 2015). This soil-borne pathogen is difficult to control because of its long persistence in the soil and wide host range (Bradley et al., 2001).

Some chemicals are effective in controlling soil-borne diseases, but they are expensive and not environmentally friendly. Recently, biological control has intensively been studied by many researchers throughout the world. Bio-inoculants containing living cells of microorganisms could be an effective alternative for chemicals in food production ( $\mathrm{O}^{\prime}$ Callaghan, 2016). Rhizosphere organisms provide an initial barrier against pathogens attacking the roots (Akhtar and Siddiqui, $2010 \mathrm{~b}$ ) and microorganisms that can grow in the rhizosphere are ideal for use as biocontrol agents. Arbuscular mycorrhizal fungi (AM fungi) associated with the roots of many crop plants can enhance plant growth and nutrient uptake (Rouphael et al., 2015) also reduce the severity of several plant diseases (Tripathi et al., 2017) and induce plant systemic resistance against root pathogens (Pérez-de-Luque et al., 2017). At present, PGPR inoculation constitutes a promising alternative strategy to reduce the use of chemical pesticides and fertilizers (Adesemoye et al., 2009 \& O'Callaghan, 2016).

However, some PGPR has a strong stimulatory impact on the growth of AM fungi (Linderman, 1997). It is worthy to mention that plant co-inoculation with either Pseudomonas fluorescens, Bacillus subtilis or Paenibacillus polymyxa can further promote the colonization of plant roots by AM fungi (Arthurson et al., 2011 \& Priyadharsini and Muthukumar, 2016). Also, support the mycorrhizal symbiosis as mycorrhization helper bacteria which stimulates root development (Garbaye, 1994 \& Choudhary et al., 2017). Likewise, the root AM fungi association constitutes the major factor influencing the community structure and the biomass of soil microorganisms leading to the establishment of the so-called mycorrhizosphere (Priyadharsini et al., 2016).

The majority of strategies for biocontrol of soil-borne plant pathogens rely on a single microbial biocontrol agent for pathogen suppression (Larkin et al., 1998). This strategy must be changed because biocontrol agents applied individually are not likely to perform consistently against all pathogens of the crop or under diverse rhizosphere and soil environmental conditions. The combination between more than

Egypt. J. Phytopathol., Vol. 46, No. 2 (2018) 
one bio-agent in a single preparation is an approach to overcoming this inconsistent performance. A combination of biocontrol agents is more likely to have a greater variety of traits responsible for the suppression of one or more pathogens and, also, is likely to have these traits expressed over a wide range of environmental conditions. Several works suggest the combination between several biocontrol agents has shown to improve the efficacy and reduce the variability of efficacy (Guetsky et al., 2002; Roberts et al., 2005 and Pertot et al., 2017).

So, the current study aims to investigate the effects of biopriming soybean seeds with Mycorrhizeen compound (mainly Glomus sp.) Paenibacillus polymyxa and Pseudomonas fluorescens either individually or in different combinations to suppress the infection by Rhizoctonia damping-off with regards to promotion of plant growth under greenhouse and field conditions.

\section{Materials and Methods}

\section{Plant materials:}

Soybean seeds (Glycine max L. Merrill) cultivar Giza 22 was obtained from the Legume Res. Dept., Field Crops Res. Inst., ARC, Giza, Egypt.

Source of the pathogen and inoculum preparation:

The fungus Rhizoctonia solani Kühn isolated from naturally infected soybean plants, showing damping-off symptoms was kindly provided by legume and forage dis. Res. Dept., Plant Path. Res. Inst., ARC, Giza, Egypt. Inoculum of R. solani was prepared according to Atwa et al., (2014).

Biotic agents: Two PGPR isolates and the bio- product Mycorrhizeen were used.

\section{A) PGPR isolates:}

Both Paenibacillus polymyxa (isolate 9D14), and Pseudomonas fluorescens were previously isolated and identified using Biolog system according to the recommended procedure of Shehata et al. (2006). P. polymyxa was activated on fresh slants of nutrient yeast dextrose agar (NYDA) medium and after $24 \mathrm{hrs}$ transferred to flasks contained $50 \mathrm{ml}$ of broth (NYDB) medium (per liter: nutrient broth $8 \mathrm{~g}$, yeast extract $5 \mathrm{~g}$ and dextrose $10 \mathrm{~g}$ ) and $P$. fluorescens was grown in flask contained King's medium B (KMB) broth (per liter: Proteose peptone 20 g, Glycerol $10 \mathrm{~mL}, \mathrm{~K}_{2} \mathrm{HPO}_{4} 1.5 \mathrm{~g}$ and $\mathrm{MgSO}_{4} 1.5 \mathrm{~g}$ ). The flasks were placed on a rotary shaker to grow at $120 \mathrm{rpm}$ for $66 \mathrm{hrs}$. at $24 \pm 1^{\circ} \mathrm{C}$.

\section{B) Mycorrhizeen:}

Mycorrhizeen formulation contains mainly endospores of Glomus sp. and its carrier free from Mycorrhizae were kindly obtained from Agric. Microbiol. Dept., Soils, Water, and Environ. Res. Inst., Agric. Res. Cent., Giza, Egypt. The carrier composed of peat moss and vermiculite $1: 1(\mathrm{w} / \mathrm{w})$ and dried at $70^{\circ} \mathrm{C}$ for $24 \mathrm{~h}$, then milled to pass through $200 \mu \mathrm{m}$ mesh sieves; $\mathrm{pH}$ of the peat moss and vermiculite was neutralized by adding calcium carbonate. 
Compatibility among bacterial strains:

The isolates of $P$. fluorescens and $P$. polymyxa were tested for their compatibility among each other following the method of Fukui et al. (1994).

Seed and soil treatments:

Apparently, healthy uniformity seeds of soybean (cv. Giza 22) were surface disinfested by immersing in sodium hypochlorite $(1 \%)$ for $2 \mathrm{~min}$, and washed several times with sterilized water, then left to dry on screen cloth with paper towel underneath to absorb the excess water at room temperature for approximately two hours.

\section{A) Biopriming treatments:}

For single treatment, $300 \mathrm{ml}$ of bacterial culture broth was used per one $\mathrm{Kg}$ of the carrier material, while for the mixed cultures, $150 \mathrm{~mL}$ of each bacterial broth was mixed with the carrier. In the case of bacterial cultures mixed with Mycorrhizeen, $300 \mathrm{~mL}$ of the bacterial culture broth in the case of single isolate or $150 \mathrm{~mL}$ from each bacterial broth in the case of mixed cultures were mixed with one $\mathrm{Kg}$ of Mycorrhizeen formulation. Healthy seeds of soybean were coated with the tested bacterial and Mycorrhizeen formulation either solely or in different combinations, using sucrose saturated solution to cover the seeds as adhesive $15 \mathrm{hrs}$. before sowing time. The coated seeds were left to air-dry on screen cloth.

\section{B) Fungicide treatment:}

Seed dressing was carried out by applying the Rizolex-T 50\% WP (Tolclofos methyl+thiram) at the recommended dose $(3 \mathrm{~g} / \mathrm{kg})$, using $1 \%$ methyl cellulose as adhesive 15 hrs. before sowing. The dressed seeds were left to dry on screen cloth.

C) Control:

The disinfected soybean seeds were coated with the peat moss and vermiculitebased formulation and the coated seeds were left to air-dry on screen cloth $15 \mathrm{hrs}$. before sowing time.

\section{Greenhouse experiments:}

The trials were carried out in the greenhouse of Plant Pathol. Res. Inst., Agric. Res. Cent., Giza. Plastic pots (25 cm in diameter) with a bottom drainage hole were sterilized by dipping in 5\% formalin solution for 15 minutes and left for one week until complete formalin evaporation. Pots were filled with steamed disinfested sandy clay soil 1:2(v/v). Soil infestation was achieved by mixing the inoculum of $R$. solani with the soil at the rate of $2 \%$ of soil weight. Sterilized un-inoculated ground sorghum grains were added to the disinfested soil at the same rate as control. The infested soil was mixed thoroughly and watered every 2 days for a week before planting to stimulate fungal growth. Five grams of Bradyrhizobium sp (rhizobium formulation), obtained from Biofert. Prod. Unit, Soils Water and Environ. Res. Inst., Agric. Res. Centre, Giza, Egypt) were added to each pot during sowing. Five seeds of pre-treated soybean seeds were sown in each pot and pots were irrigated directly. Twelve replicated pots were used for each particular treatment. All pots were irrigated when it was necessary and fertigated one month after sowing and every week until the end of the experiment to near field capacity with a $0.1 \% 15: 15: 15$ (N: $\mathrm{P}: \mathrm{K})$ fertilizer solution. The serial number (S.) and treatments are as shown in Table (1).

Egypt. J. Phytopathol., Vol. 46, No. 2 (2018) 
Table 1. Serial number (S.) and treatments

\begin{tabular}{|c|c|c|c|}
\hline S. number & Treatment & S. number & Treatment \\
\hline 1 & Mycorrhizeen & 6 & Mycorrhizeen $+P$. fluorescens \\
\hline 2 & P. polymyxa & 7 & $\begin{array}{l}\text { Mycorrhizeen }+P . \text { polymyxa } \\
+P \text {.fluorescens }\end{array}$ \\
\hline 3 & P. fluorescens & 8 & Rizolex-T \\
\hline 4 & $\begin{aligned} & \text { P. polymyxa } \\
&+ P . \text { fluorescens } \\
&\end{aligned}$ & 9 & $\begin{array}{c}\text { Untreated control } \\
\text { (R. solani infested soil) }\end{array}$ \\
\hline 5 & $\begin{array}{l}\text { Mycorrhizeen } \\
+P \text {. polymyxa }\end{array}$ & 10 & $\begin{array}{l}\text { Untreated control healthy } \\
\text { (non-infested soil) }\end{array}$ \\
\hline
\end{tabular}

Twelve plants (four replicates each of three plants) were uprooted 60 days after sowing. Roots were washed using slow running water to remove soil particles and organic debris. Then, the number of nodules per root system was counted after detaching nodules from main and secondary (lateral) roots. Shoots length was measured and cut at the soil line. Shoots, roots and nodules were placed in paper bags and oven-dried at $70^{\circ} \mathrm{C}$ for $48 \mathrm{~h}$, then weighed and the average were recorded. Nitrogen, phosphorus and potassium contents were estimated in the shoots. Where the dry plant samples were ground and prepared for wet digestion using $\mathrm{H}_{2} \mathrm{SO}_{4}$ and $\mathrm{H}_{2} \mathrm{O}_{2}$ methods as described by Page et al. (1982). The digests were then subjected to the measurement of nutrients i.e, nitrogen, phosphorus and potassium (Cottenie et al., 1982).

\section{Disease assessment:}

Disease incidence (DI) \% was determined by recording pre-emergence and postemergence damping-off, as well as the percentages of survived plants 15, 30 and 45 days after sowing respectively. Reduction or increasing \% over the infected control was also calculated according to the following formula:

$$
\text { Reduction or Increasing } \%=\frac{\text { DI of Control - DI of treatment x } 100}{\text { DI of Control }}
$$

\section{Mycorrhizae colonization of soybean root:}

The percentages of soybean root colonization by Vesicular Mycorrhizal fungus were determined 60 days after sowing. Roots staining technique was applied according to Koske and Gemma (1989). The root system was washed with tap water several times to remove the adhering soil particles. Roots were cut into small segments and cleared in potassium hydroxide $(\mathrm{KOH}) 5 \%(\mathrm{w}: \mathrm{v})$ at $90^{\circ} \mathrm{C}$ for $30 \mathrm{~min}$. After treatment with $\mathrm{KOH}$, the roots were then rinsed in several changes of water and acidified by immersing in $\mathrm{HCl}(1 \%)$. The volume of acid equal to ca $25 \mathrm{x}$ the volume of the roots was used in an overnight soak. Acidified roots were stained in an acidic glycerol solution (500 $\mathrm{ml}$ glycerol, $\left.450 \mathrm{ml} \mathrm{H}_{2} \mathrm{O}, 50 \mathrm{ml} 1 \% \mathrm{HCl}\right)$ containing $0.05 \%$ trypan blue for $20 \mathrm{~min}$ in a $90^{\circ} \mathrm{C}$ water bath. The roots detained in acidic glycerol at room temperature, in roots whose cells retain excessive stain. Root colonization levels were estimated on 5 groups of 10 root segments $(1 \mathrm{~cm}$ long) randomly chosen and examined under the light microscope for the presence of 
fungal structures. Root colonization percentage was calculated according to the following formula:

Root colonization $\%=$ No. of colonized root fragments $/$ No. of total root fragments $\times 100$.

Field experiments:

Field experiments were carried out during the summer growing season of 2017 at two locations, Giza Agric. Res. Stat., Giza governorate and Etai El-Baroud Agric. Res. Stat., El- Behira Governorate in fields known to have Rhizoctonia root rot history, in order to investigate the effect of biotic and chemical treatments on controlling damping-off diseases. The disinfected soybean seeds were treated in the same manner of the greenhouse experiment. The disinfected soybean seeds were sown in the field on May $22 \& 23,2017$ for both locations respectively. The field trial was designed in a complete randomized block with four replicates. The area of each plot was $10.5 \mathrm{~m}^{2}$ consisted of five rows; each row was $3.5 \mathrm{~m}$ length and $0.6 \mathrm{~m}$ width. The seeds were sown in hills at $20 \mathrm{~cm}$ apart on both sides of the row ridge, with one seed per hill. Eight hundred grams of Bradyrhizobium formulation (rhizobium) were mixed with approximately $50 \mathrm{~kg}$ of moistened fine sandy soil and added to field soil (feddan). All agricultural practices i.e., irrigation, fertilization, hoeing weed and pests control were carried out as the recommendation of min. of Agric. and land. Recl. for soybean.

The treatments were implemented as mentioned in Table 1 and disease incidence (DI) \% was determined as mentioned before. Random samples of ten soybean plants were collected (from the inner rows) at the harvest stage from each plot. Crop parameters i.e., plant height, number of branches, number of pods per plant, the weight of one hundred seeds and seeds yield per plant were recorded as well as seeds yield $(\mathrm{Kg}) /$ feddan were estimated.

Effect of biopriming soybean seed with biotic treatment on the activity of oxidative enzymes and phenol content:

An experiment was carried out to determine the activity of oxidative enzymes and phenolic compounds. Soybean plants were grown as mentioned before in the greenhouse experiment under biocontrol treatments and untreated control in infested soil as well as untreated control for non-infested soil (Table 1). Fifteen days after sowing, the activity of peroxidase (PO), polyphenol oxidase (PPO) and phenolic contents were determined in tissue extracts of soybean plants.

Assay of enzymes activity:

A) Assay of peroxidase (PO):

Extraction and assay of peroxidase (PO) activity were carried out according to Chakraborty \& Chatterjee (2007).

B) Assay of polyphenol oxidase (PPO):

Extraction and assay of polyphenol oxidase enzyme (PPO) were carried out according to Sadasivam \& Manickam (1996). 
Determination of phenolic compounds:

The extraction of phenolic compounds was carried out according to Sutha et al (1998). Phenolic compounds were determined using methods of analysis described by Snell \& Snell (1953).

Statistical analysis:

Completely randomized design (CRD) and randomized blocks design (RBD) were implemented in the greenhouse experiment and field experiment, respectively. The obtained data were subjected to computer statistical software (ASSISTAT) originated by Silva \& Azevedo (2009). Data analyzed using analysis of variance (ANOVA), and mean values were compared using Duncan's multiple range test at a significance level of $\mathrm{P} \leq 0.05$.

\section{Results}

1-Compatibility among bacterial strains:

Bacterial isolates of $P$. polymyxa and $P$. fluorescens were tested for their compatibility in vitro. None of the antagonistic bacteria inhibited each other, suggesting that these bacterial antagonists were compatible with each other.

2-Greenhouse experiment:

1-Effect of the bioagents and Rizolex-T on the incidence of Rhizoctonia damping-off:

Results in Table 2 show that all the treatments significantly reduced the percentages of pre- and post-emergence damping-off with significant increasing of the survived plants compared to control grown in infested soil by $R$. solani. The triple combination treatment (Mycorrhizeen plus $P$. polymyxa and $P$. fluorescens) and Rizolex-T treatment have highly reduced the pathogenesis of $R$. solani on germinated soybean seeds pre-emergence as they occurred $4 \%$ dead seeds as control healthy grown in non-infested soil. Such curative effect extended to post-emergence data and the results of survived plants after 45 days of cultivation. However, all of the biocontrol treatments were less insignificant efficiency compared with Rizolex-T and triple combination treatment. It is obvious that mixed compatible bioagents were highly effective than single bio-treatments, but they resulted in the same effect at post-emergence. 
Table 2. Effect of the tested bio-agents and Rizolex-T as seed treatments on the percentage of damping-off disease of soybean plants grown in artificially infested soil by $R$. solani under greenhouse conditions

\begin{tabular}{|c|c|c|c|c|c|c|}
\hline \multirow{3}{*}{ Treatment } & \multicolumn{4}{|c|}{ \% Damping-off } & \multirow{3}{*}{$\begin{array}{c}\% \\
\text { Survived } \\
\text { plants }\end{array}$} & \multirow{3}{*}{$\begin{array}{c}\% \\
\text { Increasing }\end{array}$} \\
\hline & \multicolumn{2}{|c|}{ Pre-emergence } & \multicolumn{2}{|c|}{ Post- emergennce } & & \\
\hline & $\begin{array}{c}\% \\
\text { Incidence }\end{array}$ & $\begin{array}{c}\% \\
\text { Reduction }\end{array}$ & $\begin{array}{c}\% \\
\text { Incidence }\end{array}$ & $\begin{array}{c}\% \\
\text { Reduction }\end{array}$ & & \\
\hline Mycorrhizeen & $12.0 \mathrm{~b}$ & 70.0 & $4.0 \mathrm{bc}$ & 66.7 & $84.0 \mathrm{ab}$ & 75.0 \\
\hline P.polymyxa & $12.0 \mathrm{~b}$ & 70.0 & $4.0 \mathrm{bc}$ & 66.7 & $84.0 \mathrm{ab}$ & 75.0 \\
\hline P. fluorescens & $12.0 \mathrm{~b}$ & 70.0 & $8.0 \mathrm{~b}$ & 33.3 & $80.0 \mathrm{~b}$ & 66.6 \\
\hline $\begin{array}{l}\text { P. polymyxa } \\
+P \text {.fluorescens }\end{array}$ & $8.0 \mathrm{~b}$ & 80.0 & $4.0 \mathrm{bc}$ & 66.7 & $88.0 \mathrm{ab}$ & 83.3 \\
\hline $\begin{array}{l}\text { Mycorrhizeen } \\
+P \text {. polymyxa }\end{array}$ & $8.0 \mathrm{~b}$ & 80.0 & $4.0 \mathrm{bc}$ & 66.7 & $88.0 \mathrm{ab}$ & 83.3 \\
\hline $\begin{array}{l}\text { Mycorrhizeen } \\
+P . \text { fluorescens }\end{array}$ & $8.0 \mathrm{~b}$ & 80.0 & $4.0 \mathrm{bc}$ & 66.7 & $88.0 \mathrm{ab}$ & 83.3 \\
\hline $\begin{array}{l}\text { Mycorrhizeen } \\
+P . \text { polymyxa } \\
+P . \text { fluorescens }\end{array}$ & $4.0 \mathrm{~b}$ & 90.0 & $4.0 \mathrm{bc}$ & 66.7 & $92.0 \mathrm{ab}$ & 91.7 \\
\hline Rizolex-T & $4.0 \mathrm{~b}$ & 90.0 & $4.0 \mathrm{bc}$ & 66.7 & $92.0 \mathrm{ab}$ & 91.7 \\
\hline $\begin{array}{l}\text { Untreated control } \\
\text { (infested soil) }\end{array}$ & $40.0 \mathrm{a}$ & 0.0 & $12.0 \mathrm{a}$ & 0.0 & $48.0 \mathrm{c}$ & 0.0 \\
\hline $\begin{array}{l}\text { Untreated control } \\
\text { (non-infested soil) }\end{array}$ & $4.0 \mathrm{~b}$ & - & $0.0 \mathrm{c}$ & - & $96.0 \mathrm{a}$ & - \\
\hline
\end{tabular}

Means in each column followed by the same letters are not significantly different using Duncan's multiple range test, $(\mathrm{p}=0.05)$.

II- Effect of the tested bioagents and Rizolex-T on some crop parameters of soybean plants grown in artificially infested soil by $R$. solani under greenhouse conditions

Results in Table 3 indicate that all treatments caused significant increases in crop parameters of soybean plants grown in infested soil with $R$. solani. For plant height, there were significant differences between all treatments and control. Meanwhile there were no significant differences among sole treatment Mycorrhizeen, $P$. polymyxa and dual treatment (Mycorrhizeen plus $P$. polymyxa), or (Mycorrhizeen plus $P$. fluorescens) treatments. The maximum height was observed for triple combination treatment (Mycorrhizeen plus $P$. polymyxa and $P$. fluorescens) followed by Rizolex-T treatment as 63.3 and $60.4 \mathrm{~cm}$ respectively, however, $P$. fluorescens as an individual treatment showed the lowest increase in plant height compared with other treatments. As for shoot dry weight, the significant maximum value was observed with triple combination treatment, healthy untreated plants, Mycorrhizeen, and dual treatment of Mycorrhizeen plus $P$. fluorescens, respectively. Meantime, the other treatments have resulted in gained values near the Rizolex-T treatment. However, for root dry weight the triple combination treatment showed the highest value. Similar effect of bioagents was obtained for both the number of nodules/plant or total dry weight $(\mathrm{mg})$ of nodules/plant. Nodulation was varied among treatments, with significant increase over infested control, the maximum 
increase was observed with triple combination treatment followed by the dual combination (Mycorrhizeen plus P. fluorescens) and Mycorrhizeen, respectively. The levels of $\mathrm{N}, \mathrm{P}$ and $\mathrm{K}$ contents were increased in all treatments compared with infected control, and the maximum increase observed with triple combination treatment.

III- Effect of the bacterial bioagents on mycorrhizae colonization of roots of soybean plants grown in artificially infested soil by Rhizoctonia solani under greenhouse conditions:

The results showed that combined treatments enhanced the percentage of colonization of mycorrhizae in the roots over Mycorrhizeen sole treatment as 18.7, 13.8 and $8.8 \%$ for the triple combination treatment (Mycorrhizeen plus $P$. polymyxa and $P$. fluorescens), the dual combination treatments (Mycorrhizeen plus $P$. fluorescens) and (Mycorrhizeen plus $P$. polymyxa), respectively.

Table 3. Effect of the tested bio-agents and Rizolex-T as seed treatments on some crop parameters and nodulation as well as nutrient contents of soybean plants grown in artificially infested soil with $R$. solani under greenhouse conditions

\begin{tabular}{|c|c|c|c|c|c|c|c|c|}
\hline \multirow[b]{2}{*}{ Treatment } & \multirow[b]{2}{*}{$\begin{array}{l}\text { Plant } \\
\text { height } \\
(\mathrm{cm})\end{array}$} & \multirow{2}{*}{$\begin{array}{c}\text { Shoot } \\
\text { dry } \\
\text { weight } \\
\text { (g/plant) }\end{array}$} & \multirow{2}{*}{$\begin{array}{c}\text { Root dry } \\
\text { weight } \\
\text { (g/plant) }\end{array}$} & \multirow[b]{2}{*}{$\begin{array}{c}\text { No. of } \\
\text { nodules/ } \\
\text { plant }\end{array}$} & \multirow{2}{*}{$\begin{array}{c}\text { Nodules } \\
\text { dry } \\
\text { weight } \\
\text { mg/plant }\end{array}$} & \multicolumn{3}{|c|}{ NPK contents } \\
\hline & & & & & & $\begin{array}{c}\mathrm{N} \\
\mathrm{mg} / \mathrm{g}\end{array}$ & $\begin{array}{c}\mathrm{P} \\
\mathrm{mg} / \mathrm{g}\end{array}$ & $\begin{array}{c}\mathrm{K} \\
\mathrm{mg} / \mathrm{g}\end{array}$ \\
\hline Mycorrhizeen & $59.9 \mathrm{~cd}$ & $4.10 \mathrm{abc}$ & $1.41 \mathrm{~b}$ & $145.3 \mathrm{~b}$ & $415.1 \mathrm{~b}$ & 3.58 & 0.301 & 2.26 \\
\hline P. polymyxa & $58.5 \mathrm{~cd}$ & $3.80 \mathrm{cde}$ & $1.30 \mathrm{~cd}$ & $131.7 \mathrm{c}$ & $394.9 \mathrm{bc}$ & 3.49 & 0.277 & 1.79 \\
\hline P. fluorescens & $55.2 \mathrm{e}$ & $3.67 \mathrm{de}$ & $1.27 \mathrm{~d}$ & $130.3 \mathrm{c}$ & $381.4 \mathrm{bc}$ & 3.53 & 0.285 & 1.87 \\
\hline $\begin{array}{l}\text { P. polymyxa+ } \\
\text { P. fluorescens }\end{array}$ & $57.8 \mathrm{~d}$ & $3.90 \mathrm{de}$ & $1.30 \mathrm{~cd}$ & $128.0 \mathrm{c}$ & $390.7 \mathrm{bc}$ & 3.62 & 0.291 & 1.89 \\
\hline $\begin{array}{l}\text { Mycorrhizeen }+ \\
\text { P. polymyxa }\end{array}$ & $58.1 \mathrm{~cd}$ & $3.65 \mathrm{e}$ & $1.31 \mathrm{~cd}$ & $134.7 \mathrm{c}$ & $384.5 b c$ & 3.62 & 0.318 & 2.20 \\
\hline $\begin{array}{l}\text { Mycorrhizeen } \\
+P \text {.fluorescens }\end{array}$ & $59.3 \mathrm{~cd}$ & $4.10 \mathrm{abc}$ & $1.39 \mathrm{bc}$ & $149.0 \mathrm{~b}$ & $420.1 \mathrm{~b}$ & 3.76 & 0.316 & 2.40 \\
\hline $\begin{array}{l}\text { Mycorrhizeen }+ \\
\text { P. polymyxa }+ \\
\text { P. fluorescens }\end{array}$ & $62.3 \mathrm{~b}$ & $4.40 \mathrm{a}$ & $1.76 \mathrm{a}$ & $175.3 \mathrm{a}$ & $460.1 \mathrm{a}$ & 3.90 & 0.332 & 2.66 \\
\hline Rizolex $-\mathrm{T}$ & $60.4 \mathrm{bc}$ & $3.90 \mathrm{cde}$ & $1.40 \mathrm{~b}$ & $128.5 \mathrm{~d}$ & $340.7 \mathrm{c}$ & 3.45 & 0.284 & 2.17 \\
\hline $\begin{array}{l}\text { Untreated control } \\
\text { (infested soil) }\end{array}$ & $35.3 \mathrm{f}$ & $2.20 \mathrm{f}$ & $0.76 \mathrm{e}$ & $32.7 \mathrm{e}$ & $82.2 \mathrm{~d}$ & 2.80 & 0.237 & 1.58 \\
\hline $\begin{array}{l}\text { Untreated control } \\
\text { (non-infested soil) }\end{array}$ & $65.2 \mathrm{a}$ & $4.20 \mathrm{ab}$ & $1.35 \mathrm{bc}$ & $181.0 \mathrm{a}$ & 471.9 a & 3.54 & 0.286 & 2.28 \\
\hline
\end{tabular}

Means in each column followed by the same letters are not significantly different using Duncan's multiple range test, $(p=0.05)$. 
3-Field experiments

I- Effect of the bioagents and Rizolex-T on the incidence of soybean damping-off disease

Results in Table 4 indicate that all treatments significantly decreased the percentage of pre and post-emergence damping-off as well as survived plants compared with untreated control in the two locations. However, the triple combination treatment (Mycorrhizeen plus $P$. polymyxa, and $P$. fluorescens) resulted in high values of disease control near to that obtained with Rizolex-T treatment. Such an effect was true for pre and post-emergence as well as the percentage of survived plants. It is obvious that mixed compatible bioagents were highly effective for reducing pre-emergence damping-off than single bio-treatments, but they resulted in the same effect at post-emergence. It is evidently observed that values of post-emergence damping-off were clearly lower than those of pre-emergence damping-off in all treatments such effect was true in the untreated control treatment.

II- Effect of the tested bioagents and Rizolex-T on some crop parameters and yield of soybean plants

Results in Table 5 exhibited that under field conditions all treatments significantly showed stimulatory effect for crop parameters of soybean plants and yield compared to the untreated control treatment in the two locations when treated separately or in combination.

Plant height:

Among all treatments, the triple combination treatment (Mycorrhizeen plus $P$. polymyxa, and P. fluorescens) as well as Rizolex-T treatment showed significantly the maximal effect at Giza and Etai-El-Baroud Rese. Stat. It is obvious that there was no significant difference among all the dual combination treatments in two locations.

The number of branches:

At two locations, all treatments significantly increased the number of branches as compared with the untreated control, and the maximum increase was recorded with the triple combination treatment. Also, there were no significant differences between the dual combination treatments and Rizolex-T treatment.

Number of pods per plant:

The number of pods per plant was significantly increased in all treatments compared with untreated control. The maximum increase was recorded with the triple combination treatment, Rizolex-T treatment and the dual combination treatment (Mycorrhizeen plus P. fluorescens) respectively with no significant difference among them at two locations.

Seed weight per plant:

In the two locations, all treatments significantly increased seed weight per plants as compared with untreated control. The maximum figures were recorded with the triple combination treatment as well as Rizolex-T treatment. There is no significant difference among all the dual combination treatments at two locations. 
Table 4. Effect of the tested bio-agents and Rizolex- $T$ as seed treatments on the percentage of soybean damping-off under field conditions (natural infection) at Giza Agric. Res. Stat. (A) and Etai El-Baroud Agric. Res. Stat. (B) during the summer growing season 2017

\begin{tabular}{|c|c|c|c|c|c|c|}
\hline \multirow[b]{3}{*}{ Treatment } & \multicolumn{4}{|c|}{$\%$ Damping-off } & \multirow{3}{*}{$\begin{array}{c}\% \text { Survived } \\
\text { Plants }\end{array}$} & \multirow{3}{*}{$\begin{array}{c}\% \\
\text { Increasing }\end{array}$} \\
\hline & \multicolumn{2}{|c|}{ Pre-emergence } & \multicolumn{2}{|c|}{ Post- emergence } & & \\
\hline & $\begin{array}{c}\% \\
\text { Incidence }\end{array}$ & $\begin{array}{c}\% \% \\
\text { Reduction }\end{array}$ & $\begin{array}{c}\% \\
\text { Incidence }\end{array}$ & $\begin{array}{c}\% \% \\
\text { Reduction }\end{array}$ & & \\
\hline \multicolumn{7}{|c|}{ (A): Giza Res. Stat. } \\
\hline Mycorrhizeen & $10.8 \mathrm{de}$ & 66.9 & $2.3 \mathrm{~b}$ & 60.3 & $86.9 \mathrm{c}$ & 41.4 \\
\hline P. polymyxa & $14.5 \mathrm{bc}$ & 55.7 & $2.3 \mathrm{~b}$ & 60.3 & $83.2 \mathrm{de}$ & 35.3 \\
\hline P. fluorescens & $16.1 \mathrm{~b}$ & 50.8 & $2.3 \mathrm{~b}$ & 60.3 & $81.6 \mathrm{e}$ & 32.7 \\
\hline $\begin{array}{l}P . \text { polymyxa } \\
+P \text {.fluorescens }\end{array}$ & $13.5 \mathrm{bcd}$ & 58.7 & $1.9 \mathrm{~b}$ & 67.2 & $84.6 \mathrm{~cd}$ & 37.6 \\
\hline $\begin{array}{l}\text { Mycorrhizeen } \\
+P \text { polymyxa }\end{array}$ & $11.5 \mathrm{cde}$ & 64.8 & $3.3 \mathrm{~b}$ & 43.1 & $85.2 \mathrm{~cd}$ & 38.5 \\
\hline $\begin{array}{l}\text { Mycorrhizeen } \\
+P \text { fluorescens }\end{array}$ & $10.3 \mathrm{de}$ & 68.5 & $3.5 \mathrm{~b}$ & 39.7 & $86.2 \mathrm{bc}$ & 40.2 \\
\hline $\begin{array}{l}\text { Mycorrhizeen } \\
+P \text {.polymyxa } \\
+P . \text { fluorescens }\end{array}$ & $6.1 \mathrm{f}$ & 81.3 & $2.5 \mathrm{~b}$ & 57.0 & $91.4 \mathrm{ab}$ & 48.6 \\
\hline Rizolex-T & $5.8 \mathrm{f}$ & 82.3 & $2.3 \mathrm{~b}$ & 60.3 & $91.9 \mathrm{a}$ & 49.4 \\
\hline Untreated control & $32.7 \mathrm{a}$ & $\begin{array}{l}0.0 \\
\end{array}$ & $5.8 \mathrm{a}$ & 0.0 & $61.5 \mathrm{f}$ & 0.0 \\
\hline \multicolumn{7}{|c|}{ (B): Etai Elbaroud Res. Stat. } \\
\hline Mycorrhizeen & $9.5 \mathrm{~d}$ & 72.1 & $2.9 \mathrm{~b}$ & 53.2 & $87.6 \mathrm{bc}$ & 46.7 \\
\hline P.polymyxa & $15.3 \mathrm{~b}$ & 55.1 & $1.7 \mathrm{~b}$ & 72.6 & $83.0 \mathrm{~d}$ & 39.0 \\
\hline P. fluorescens & $15.6 \mathrm{~b}$ & 54.3 & $1.5 \mathrm{~b}$ & 75.8 & $82.9 \mathrm{~d}$ & 38.9 \\
\hline $\begin{array}{l}P . \text { polymyxa } \\
+P . \text { fluorescens }\end{array}$ & $11.8 \mathrm{c}$ & 65.4 & $2.5 \mathrm{~b}$ & 59.7 & $85.7 \mathrm{~cd}$ & 43.6 \\
\hline $\begin{array}{l}\text { Mycorrhizeen } \\
+P \text {. polymyxa }\end{array}$ & $11.7 \mathrm{c}$ & 65.7 & $1.5 \mathrm{~b}$ & 75.8 & $86.8 \mathrm{bc}$ & 45.4 \\
\hline $\begin{array}{l}\text { Mycorrhizeen } \\
+P \text { fluorescens }\end{array}$ & $10.3 \mathrm{~cd}$ & 69.8 & $2.5 \mathrm{~b}$ & 59.7 & $87.2 \mathrm{bc}$ & 46.0 \\
\hline $\begin{array}{l}\text { Mycorrhizeen } \\
+P . \text { polymyxa } \\
+P . \text { fluorescens }\end{array}$ & $5.5 \mathrm{e}$ & 83.9 & $2.8 \mathrm{~b}$ & 54.8 & $91.7 \mathrm{ab}$ & 53.6 \\
\hline Rizolex-T & $5.9 \mathrm{e}$ & 82.7 & $2.1 \mathrm{~b}$ & 66.1 & $92.0 \mathrm{a}$ & 54.1 \\
\hline Untreated control & $34.1 \mathrm{a}$ & 0.0 & $6.2 \mathrm{a}$ & 0.0 & $59.7 \mathrm{e}$ & 0.0 \\
\hline
\end{tabular}

Means in each column followed by the same letters are not significantly different using Duncan's multiple range test, $(\mathrm{p}=0.05)$.

The weight of one hundred seed:

In the two locations, all treatments significantly increased the weight of one hundred seed as compared with untreated control. The maximum increase was recorded with the triple combination and Rizolex-T treatments. Also, there is no significant difference among all the dual combination treatments at two locations. However, the lowest figure was observed with $P$. fluorescens. 
Seed yield:

At Giza Res. Stat., the maximum increase in seed yield was recorded with the triple combination followed by Rizolex-T and the dual combination (Mycorrhizeen plus $P$. fluorescens) treatments, respectively. While in the Etai-El-Baroud Res. Stat. the maximum increase was noticed with Rizolex-T treatment followed by the triple combination treatment.

Table 5. Effect of the tested bio-agents and Rizolex-T as seed treatments on some cropparameters of soybean plants under field conditions (natural infection) at Giza Agric. Res. Stat. (A) and Etai El-Baroud Agric. Res. Stat. (B) during the summer growing season 2017

\begin{tabular}{|c|c|c|c|c|c|c|}
\hline Treatment & $\begin{array}{l}\text { Plant } \\
\text { height } \\
(\mathrm{cm})\end{array}$ & $\begin{array}{c}\text { No. of } \\
\text { branches/ } \\
\text { plant } \\
\end{array}$ & $\begin{array}{l}\text { No. of } \\
\text { pods/ } \\
\text { plant }\end{array}$ & $\begin{array}{c}\text { Seed } \\
\text { weight/ } \\
\text { plant }(g)\end{array}$ & $\begin{array}{c}100 \text { seed } \\
\text { weight } \\
(\mathrm{g})\end{array}$ & $\begin{array}{c}\text { Seed } \\
\text { yield } \\
(\mathrm{kg} / \mathrm{fed})\end{array}$ \\
\hline \multicolumn{7}{|c|}{ (A): Giza Res. Stat. } \\
\hline Mycorrhizeen & $90.0 \mathrm{bc}$ & $3.7 \mathrm{bc}$ & $74.3 \mathrm{abcd}$ & $30.1 \mathrm{bc}$ & $18.2 \mathrm{bc}$ & $1510 \mathrm{bc}$ \\
\hline P. polymyxa & $87.0 \mathrm{~cd}$ & $3.3 \mathrm{c}$ & $71.8 \mathrm{~cd}$ & $28.7 \mathrm{bc}$ & $18.2 \mathrm{bc}$ & $1430 \mathrm{~d}$ \\
\hline P. fluorescens & $85.7 \mathrm{~d}$ & $3.4 \mathrm{c}$ & $67.8 \mathrm{~d}$ & $27.9 \mathrm{c}$ & $17.6 \mathrm{c}$ & $1272 \mathrm{e}$ \\
\hline $\begin{array}{l}P . \text { polymyxa } \\
+P \text {. fluorescens }\end{array}$ & 88.7 bcd & $4.0 \mathrm{ab}$ & $77.4 \mathrm{abc}$ & $29.9 \mathrm{bc}$ & $18.3 \mathrm{bc}$ & $1408 \mathrm{~cd}$ \\
\hline $\begin{array}{l}\text { Mycorrhizeen } \\
+P . \text { polymyxa }\end{array}$ & $90.3 \mathrm{bc}$ & $3.9 \mathrm{~b}$ & $72.5 \mathrm{bcd}$ & $30.5 \mathrm{~b}$ & $18.3 \mathrm{bc}$ & $1384 \mathrm{de}$ \\
\hline $\begin{array}{l}\text { Mycorrhizeen } \\
+P \text { fluorescens }\end{array}$ & $91.7 \mathrm{~b}$ & $4.1 \mathrm{ab}$ & $77.8 \mathrm{abc}$ & $30.8 \mathrm{~b}$ & $18.6 \mathrm{~b}$ & $1582 \mathrm{ab}$ \\
\hline $\begin{array}{l}\text { Mycorrhizeen } \\
+P \text {. polymyxa } \\
+P \text {.fluorescens }\end{array}$ & 97.9 a & $4.5 \mathrm{a}$ & $81.0 \mathrm{a}$ & $34.0 \mathrm{a}$ & $19.1 \mathrm{a}$ & $1643 \mathrm{a}$ \\
\hline Rizolex -T & $95.9 \mathrm{a}$ & $4.0 \mathrm{ab}$ & $79.8 \mathrm{ab}$ & $33.2 \mathrm{a}$ & $18.7 \mathrm{ab}$ & $1630 \mathrm{a}$ \\
\hline Untreated control & $69.4 \mathrm{e}$ & $2.8 \mathrm{~d}$ & $38.0 \mathrm{e}$ & $23.7 \mathrm{~d}$ & $15.6 \mathrm{~d}$ & $968 \mathrm{f}$ \\
\hline \multicolumn{7}{|c|}{ (B): Etai Elbaroud Res. Stat. } \\
\hline Mycorrhizeen & $94.8 \mathrm{ab}$ & $3.8 \mathrm{bc}$ & $73.0 \mathrm{bc}$ & $30.9 \mathrm{~b}$ & $18.4 \mathrm{ab}$ & $1483 \mathrm{c}$ \\
\hline P. polymyxa & $89.7 \mathrm{bc}$ & $3.5 \mathrm{~cd}$ & $65.8 \mathrm{de}$ & $28.7 \mathrm{~cd}$ & $17.8 \mathrm{bc}$ & $1342 \mathrm{de}$ \\
\hline P.fluorescens & $86.8 \mathrm{c}$ & $3.4 \mathrm{~d}$ & $63.0 \mathrm{e}$ & $27.5 \mathrm{~d}$ & $17.4 \mathrm{~cd}$ & $1281 \mathrm{e}$ \\
\hline $\begin{array}{l}P . \text { polymyxa } \\
+P . \text { fluorescens }\end{array}$ & $92.5 \mathrm{bc}$ & $3.9 \mathrm{bc}$ & 68.2 cde & $30.3 \mathrm{bc}$ & $17.7 \mathrm{bc}$ & $1395 \mathrm{~d}$ \\
\hline $\begin{array}{l}\text { Mycorrhizeen } \\
+P \text {. polymyxa }\end{array}$ & $94.1 \mathrm{~b}$ & $4.2 \mathrm{ab}$ & $69.0 \mathrm{~cd}$ & $30.8 \mathrm{bc}$ & $18.2 \mathrm{abc}$ & $1361 \mathrm{de}$ \\
\hline $\begin{array}{l}\text { Mycorrhizeen } \\
+P \text {.fluorescens }\end{array}$ & $91.4 \mathrm{bc}$ & $4.2 \mathrm{ab}$ & $74.6 \mathrm{ab}$ & $32.0 \mathrm{~b}$ & $18.2 \mathrm{abc}$ & $1517 \mathrm{bc}$ \\
\hline $\begin{array}{l}\text { Mycorrhizeen } \\
+P \text {. polymyxa } \\
+P \text {.fluorescens }\end{array}$ & $98.7 \mathrm{a}$ & $4.3 \mathrm{a}$ & $78.5 \mathrm{a}$ & $34.7 \mathrm{a}$ & $18.6 \mathrm{a}$ & $1603 \mathrm{ab}$ \\
\hline Rizolex-T & $96.9 \mathrm{a}$ & $4.0 \mathrm{ab}$ & $76.5 \mathrm{ab}$ & $34.1 \mathrm{a}$ & $18.4 \mathrm{ab}$ & $1625 \mathrm{a}$ \\
\hline Untreated control & $67.5 \mathrm{~d}$ & $2.8 \mathrm{e}$ & $36.3 \mathrm{f}$ & $23.9 \mathrm{e}$ & $14.5 \mathrm{e}$ & $1005 \mathrm{f}$ \\
\hline
\end{tabular}

Means in each column followed by the same letters are not significantly different using Duncan's multiple range test, $(p=0.05)$ 
From the results, it could be noticed that no significant differences between the triple combination treatment and Rizolex-T treatment in all growth parameters under study at two locations. However, there were no significant differences between the two dual combination treatments ( $P$. polymyxa plus $P$. fluorescens), and (Mycorrhizeen plus $P$. polymyxa) in all crop parameters under study at two locations. Meanwhile, no significant differences were found between the two sole treatments with $P$. polymyxa or $P$. fluorescens in all crop parameters under study at two locations with exception of seed yield ( $\mathrm{kg} /$ feddan) at Giza Res. Stat.

4- Effect of the tested bioagents as seed treatments on the activity of oxidative enzymes and content of phenolic compounds:

a. Activity of oxidative enzymes:

Data in Table 6 showed increases of peroxidase (PO) and polyphenol oxidase (PPO) in treated soybean plants with different bioagent treatments over the untreated control plants sown in infested soil with $R$. solani. The maximum increase was recorded with the triple combination treatment and this was true for both enzymes. However, the lowest increase was observed with $P$. fluorescens as a sole treatment for the two enzymes.

Table 6. Effect of the tested bio-agents as seed treatments on the activity of peroxidase and polyphenol oxidase in soybean plants grown in artificially infested soil by $R$. solani under greenhouse condition

\begin{tabular}{|c|c|c|c|c|}
\hline \multirow[t]{2}{*}{ Treatment } & \multicolumn{2}{|c|}{$\begin{array}{c}\text { Peroxidase activity } \\
\text { (absorbance at } 430 \mathrm{~nm}) \\
(\text { Enzyme unit } / \mathrm{mg} \text { protein } / \mathrm{min})\end{array}$} & \multicolumn{2}{|c|}{$\begin{array}{l}\text { Polyphenol oxidase activity } \\
\text { (absorbance at } 495 \mathrm{~nm} \text { ) } \\
\text { (Enzyme unit } / \mathrm{mg} \\
\text { protein } / \mathrm{min} \text { ) }\end{array}$} \\
\hline & Activity & $\begin{array}{l}\% \text { increasing } \\
\text { over control }\end{array}$ & Activity & $\begin{array}{l}\% \text { increasing } \\
\text { over control }\end{array}$ \\
\hline Mycorrhizeen & 2.052 & 21.3 & 0.215 & 58.1 \\
\hline P. polymyxa & 2.131 & 25.9 & 0.221 & 62.5 \\
\hline P. fluorescens & 1.997 & 18.0 & 0.193 & 41.9 \\
\hline $\begin{array}{l}\text { P. polymyxa } \\
+P \text {. fluorescens }\end{array}$ & 2.216 & 30.9 & 0.232 & 70.6 \\
\hline $\begin{array}{l}\text { Mycorrhizeen } \\
+P \text {. polymyxa } \\
\end{array}$ & 2.672 & 57.9 & 0.245 & 80.1 \\
\hline $\begin{array}{l}\text { Mycorrhizeen } \\
+P \text {.fluorescens }\end{array}$ & 2.531 & 49.6 & 0.253 & 86.0 \\
\hline $\begin{array}{l}\text { Mycorrhizeen } \\
+P \text {. polymyxa } \\
+P \text {.fluorescens } \\
\end{array}$ & 2.736 & 61.7 & 0.321 & 136.0 \\
\hline $\begin{array}{l}\text { Untreated control } \\
\text { (infested soil) }\end{array}$ & 1.692 & 0.0 & 0.136 & 0.0 \\
\hline $\begin{array}{l}\text { Untreated control } \\
\text { (non-infested soil) }\end{array}$ & \multicolumn{2}{|c|}{1.256} & \multicolumn{2}{|c|}{0.129} \\
\hline
\end{tabular}




\section{b. Phenol content:}

Data in Table 7 indicated that all treatments enhanced the total phenol content in soybean plants compared with untreated control. The maximum increase was recorded with the triple combination treatment followed by the two dual treatments of the Mycorrhizeen. However, the lowest increase was noted with $P$. fluorescens and $P$. polymyxa as a sole treatment. The same results were observed for the contents of free phenol. Meanwhile, the fewer figure for total phenol, free phenol, and conjugated phenol was observed with $P$. fluorescens treatments.

Table 7. Effect of the tested bio-control agents as seed treatments on the levels of phenolic compounds in soybean plants grown in artificially infested soil by $R$. solani under greenhouse condition

\begin{tabular}{|l|c|c|c|c|c|c|}
\hline \multirow{2}{*}{ Treatment } & \multicolumn{5}{|c|}{ Phenolic contents (catechol equivalents mg/g fresh weight) } \\
\cline { 2 - 8 } & $\begin{array}{c}\text { Total } \\
\text { phenols }\end{array}$ & $\begin{array}{c}\text { \% Increase } \\
\text { over control }\end{array}$ & $\begin{array}{c}\text { Free } \\
\text { phenols }\end{array}$ & $\begin{array}{c}\text { \% Increase } \\
\text { over control }\end{array}$ & $\begin{array}{c}\text { Conjugated } \\
\text { phenols }\end{array}$ & $\begin{array}{c}\text { \% Increase } \\
\text { over control }\end{array}$ \\
\hline Mycorrhizeen & 3.325 & 48.3 & 1.693 & 16.5 & 1.632 & 105.8 \\
\hline P.polymyxa & 3.123 & 39.3 & 1.532 & 5.4 & 1.591 & 101.6 \\
\hline P. fluorescens & 2.857 & 27.4 & 1.493 & 2.7 & 1.364 & 72.9 \\
\hline $\begin{array}{l}P . \text { polymyxa } \\
+P . \text { fluorescens }\end{array}$ & 3.456 & 54.1 & 1.792 & 23.3 & 1.664 & 110.9 \\
\hline $\begin{array}{l}\text { Mycorrhizeen } \\
+P . \text { polymyxa }\end{array}$ & 3.543 & 58.0 & 1.893 & 30.2 & 1.65 & 109.1 \\
\hline $\begin{array}{l}\text { Mycorrhizeen } \\
+P . \text { fluorescens }\end{array}$ & 3.573 & 59.4 & 1.935 & 33.2 & 1.638 & 107.6 \\
\hline $\begin{array}{l}\text { Mycorrhizeen } \\
+P . \text { polymyxa } \\
+ \text { P.fluorescens }\end{array}$ & 3.828 & 70.7 & 2.135 & 46.9 & 1.693 & 114.6 \\
\hline $\begin{array}{l}\text { Untreated control } \\
\text { (infested soil) }\end{array}$ & 2.242 & 0.0 & 1.453 & 0.0 & 0.789 & 0.0 \\
\hline $\begin{array}{l}\text { Untreated control } \\
\text { (non-infested soil) }\end{array}$ & 1.523 & & 1.259 & & 0.323 \\
\hline
\end{tabular}

\section{D i s c us s i o n}

The wide host range exhibited by $R$. solani complicates management schemas because there are no commercial soybean cultivars resistant to this pathogen (Bradley et al., 2001). However, the Intensive use of agrochemicals has been a subject of public concern because of harmful effects on the natural resources such as water and potential carcinogenicity of some chemicals (Carvalho, 2017). So, recent research priorities preferred disease control programs that are safe and compatible with sustainable agriculture. The present study demonstrates that not only the seed treatment with Rizolex-T decreased the disease incidence significantly but also treated seeds with $P$. polymyxa, $P$. fluorescens and Mycorrhizeen formulation (mainly Glomus sp.) in combination as dual or triple inoculation decreased significantly the severity of damping-off disease of soybean plants under greenhouse and field conditions. In this respect, $P$. fluorescens isolates which non-pathogenic saprophytes that colonize soil and plant surface environments are effective for the management of soil-borne disease (Bakker et al., 2007 \& Susilowati et al., 2011). 
This action believed to be related to: (1) competition for nutrients (Sharma, 2006); (2) produce a wide variety of antimicrobial metabolites such as the antibiotic 2,4diacetyl phloroglucinol and pyrrolnitrin (Zhang et al., 2016); cyclic lipopeptides (Michelsen et al., 2015); (3) iron-chelating siderophores (Gupta and Gopal, 2008); (4) hydrolytic enzymes from bacteria such as chitinase, $\beta$-1,3-glucanase, and protease (Solanki et al., 2014) and (5) its induction of systemic resistance (Bakker et al., 2007).

However, $P$. polymyxa is known for its ability to produce antimicrobial compounds that suppress plant pathogens in the soil such as fusaricidins (Beatty \& Jensen, 2002); polymyxins and lantibiotics (He et al., 2007). Meanwhile, hydroxamate-type siderophores have been detected in cultures of $P$. polymyxa SQR21, in the late log phase (Raza and Shen, 2010). Also, P. polymyxa can help to control phytopathogens by triggering a hypersensitive defensive response of the plant, known as induced systemic resistance (Atwa et al., 2014).

Additionally, biocontrol effects of AM fungi on root pathogens have been previously reported (Borowicz, 2001), the AMF play an important role in the management of plant pathogens, such as Rhizoctonia solani (Yao et al., 2002) and Macrophomina phaseolina (Akhtar and Siddiqui, 2010 a). This action may be due to several mechanisms, for example, competition for space and nutrients with soilborne pathogens within the mycorrhizosphere and the host roots. (Azcón-Aguilar and Barea, 1996); activation of plant defense mechanisms against soil-borne pathogens (Bagyaraj, 2006) by modification of cell wall, production of antimicrobial compounds and altered rhizosphere microflora (Sampangi and Bagyaraj 1989; Azcón-Aguilar and Barea, 1996). The antagonistic interaction between AMF with various soilborne plant pathogens is the reason for their use as biocontrol agents. However, a synergistic or additive control of pathogen impact on plant growth by dual inoculation of AM fungi with rhizobacteria showing biocontrol properties (Barea et al., 2005).

A relational trend for the effect of bioagents on suppression of soybean damping off, that was the increase in activities of peroxidase (PO) and polyphenol oxidase (PPO) enzymes in samples of treated plants. Here again, combining bio-treatments resulted in the highest enzymatic activity which was coincided with suppression of $R$. solani pathogenesis to soybean seedlings in soil. Wang et al., (2000) stated that the increase in oxidases activity in plants was related to increased plant resistance against infection by many diseases. Among the proteins induced during plant defense and playing a key role in several metabolic responses, plant peroxidases are well known. It is widely known that peroxidases play a central role in host plant defences against necrotrophic or biotrophic pathogens (Van Loon et al., 2006). Moreover, peroxidases are involved in phytoalexin synthesis, and the production of reactive oxygen species (ROS) that have antifungal properties (Almagro et al., 2009).

Meantime, polyphenol oxidases or tyrosinases (PPO) are able to oxidize the ortho-diphenolic compounds to o-quinones (antimicrobial compounds). Quinones are effective inhibitors of SH group of enzymes which may inhibit the pathogens (Goodman et al., 1967). Also, several studies report a positive correlation between 
PPO expression and resistance/tolerance to biotic stresses (Taranto et al., 2017). In fact, Polyphenol oxidases may act through: (1) direct toxicity of quinones; (2) reduced bioavailability and alkylation of cellular proteins to the pathogen; (3) crosslinking of quinones with protein or other phenolic compounds to form physical barriers; and (4) the production of reactive oxygen species (ROS) (Yoruk and Marshall, 2003).

Accordingly, the total phenolic compounds content of soybean treated plants with the tested bioagents and sown in infested soil with $R$. solani was remarkably increased over untreated control plants. Such data are related to the recorded increase in oxidases in the same treatments. The highest increase in phenolic compounds was recorded with the triple combined bioagents. In this regard, the role of phenolic compounds in disease resistance was postulated by Nicholson and Hammerschmidt (1992). They indicated that phenols are oxidized to quinones or semi-quinones which are more toxic and play a great role as antimicrobial substances on the fungal pathogens (Farkas and Kiraly, 1962).

As for the impact of the tested bioagents on the nodulation of soybean plants compared with untreated control plants grown in $R$. solani infested soil, different bioagent treatments resulted in a significant increase in nodules number and nodules dry weight when co-inoculated with B. japonicum. Bai et al. (2003) stated that coinoculation of B. japonicum with Bacillus strains resulted in the greatest increase in the number of nodules, nodules weight, shoot and root weight and grain yield in soybean plants. Silva et al. (2007) found nodulation stimulus in Vigna unguiculata co-inoculated with Bradyrhizobium sp. and P. polymyxa. Nishijima et al. (1988) reported that $P$. fluorescens can increase the nodulation ability of $B$. japonicum, preincubation of $B$. japonicum and $P$. fluorescens before inoculation further enhanced the level of nodulation of soybean. Sayeed and Siddiqui (2008) reported that AM fungus can coexist along with root nodule bacterium without exhibiting adverse effects on each other, and it could be utilized as a biocontrol agent to control most of the soil-borne disease. Generally, legumes such as soybean can form symbiotic associations with both rhizobia and AM fungi (Lisette et al., 2003). The encouragement of shoots and roots dry weight caused by the bioagents treatments have come back on seeds yield per feddan in the field, so a clear increase was obtained with all bioagents and Rizolex-T over the untreated control. In this respect, the possible explanations for growth promotion by $P$. polymyxa that it produces many plant growth stimulators, including auxin as indole 3-acetic acid (da Mota et al., 2008); cytokinin and increased P-uptake (Timmusk et al., 1999). Also, Paenibacillus may be capable of stimulating mycorrhizal colonization as in sorghum (Budi et al., 1999). On the other hand, the increase in $\mathrm{P}$ content in plants coinoculated with P. fluorescens and G. mosseae was achieved (Gamalero et al., 2004). Previously, phosphate solubilizing Pseudomonas sp. was observed from the rhizoplane of rice (Islam et al., 2007). Bapiri et al. (2012) notified zinc solubilization in P. aeruginosa and $P$. fluorescens. Meanwhile, the most frequently documented response to AM colonization is an increase in phosphorus nutrition of the host plant, which would consequently be more vigorous and more resistant to pathogen invasion (St-Arnaud and Elsen, 2005 and Toussaint et al., 2008). 
There was an increase in NPK contents due to the treatments over the untreated control in the greenhouse experiment. High levels of $\mathrm{N}$ and $\mathrm{K}$ were found in single AM fungi inoculated plants, and this is in line with previous studies (Toussaint et al., 2004 and Mortimer et al., 2009) by enhancing biological $\mathrm{N}$ fixation. Also, AM symbiosis resulted in more vigorous growth of plants and plants may be more resistant or tolerant to pathogen attacks (Linderman, 1994). Also, Jäderlund et al. (2008) reported that dual inoculation of Paenibacillus with AM fungi stimulated growth and exerted strong stimulatory effects on AM colonization and promoted mycorrhizal root colonization. The AM symbiosis enhances the feeding of mineral nutrients to the plant, particularly the ionic forms nutrient that has poor mobility or those existing in low concentrations in the soil solution. This mainly applies to phosphate, ammonium, zinc, and copper. The AM association also upgrades plant soundness through increased defense against soil-borne microbial pathogens in the bioremediation of polluted soils (Barea et al., 2005). Based on the fact that each biocontrol agent may have a different mechanism of action to fight the pathogen, so the use of a combination of biocontrol agents expects to accomplish better results. The combined use of Bacillus pumilus, Pseudomonas alcaligenes and Glomus intraradices increases the availability of $\mathrm{N}$ and $\mathrm{P}$ which may have an adverse effect on pathogens, improves root growth than individual application (Akhtar and Siddiqui, 2008). So, Dwivedi et al. (2009) found that antifungal compounds like phenazine and di-acetyl-phloroglucinol (DAPG) produced by P. fluorescens has a positive influence on mycorrhizal colonization as well as soybean growth parameters. Xavier and Germida (2003) reported that PGPR are known to enhance AM fungal growth and root colonization. Also, high NPK concentrations were observed when inoculated plants with PGPR, Mycorrhizae which allow for increased shoot growth as well as greater above-ground biomass (Singh et al., 2012). Moreover, Souchie et al. (2003) reported the dual application of AMF and PGPR inhibited the pathogen more efficiently and reduced infections.

\section{Ref e r e n e s}

Adesemoye, A.O.; Torbert, H.A. and Kloepper, J.W. 2009. Plant growth-promoting rhizobacteria allow reduced application rates of chemical fertilizers. Microb. Ecol., 58: 921-929.

Akhtar, M.S. and Siddiqui, Z.A. 2008. Glomus intraradices, Pseudomonas alcaligenes, Bacillus pumilus as effective biocontrol agents for the root-rot disease complex of chickpea (Cicer arietinum L.). J. Gen. Plant Pathol., 74: 53-60.

Akhtar, M.S. and Siddiqui, Z.A. 2010a. Effects of AM fungi on the plant growth and root rot disease of chickpea. Am-Eurasian J. Agric. Environ. Sci., 8: 544-549.

Akhtar, M.S. and Siddiqui, Z.A. 2010b. Role of Plant Growth Promoting Rhizobacteria. In Biocontrol of Plant Diseases and Sustainable Agriculture. In: Plant Growth and Health Promoting Bacteria (Maheshwari, D. K. ed.). Springer-Verlag Berlin Heidelberg, pp 157-195. 
Almagro, L.; Gómez Ros, L.V.; Belchi-Navarro, S.; Bru, R.; Ros Barceló, A. and Pedreño. M.A. 2009. Class III peroxidases in plant defence reactions. J. Exp. Bot., 60: 377-390.

Arthurson, V.; Hjort, K.; Muleta, D.; Jaderlund, L. and Granhall, U. 2011. Effects on Glomus mosseae root colonization by Paenibacillus polymyxa and Paenibacillus brasilensis strains as related to soil P-availability in winter wheat. Appl. and Environ. Soil Sci., 2011. Article ID: 298097, 9 p.

Atwa, Marwa A.M.; Shehata, S.T. and Rahhal, M.M.H. 2014. Induction of resistance against soybean damping-off caused by Rhizoctonia solani. Egypt. J. Phytopathol., 42 (2): 137-158.

Azcón-Aguilar, C. and Barea, J.M. 1996. Arbuscular mycorrhizas and biological control of soil-borne plant pathogens - an overview of the mechanisms involved. Mycorrhiza, 6: 457-464.

Bagyaraj, D.J. 2006. Current Status of VAM as Biocontrol Agents for the Management of Plant Diseases. In: Current Status of Biological Control of Plant Diseases Using Antagonistic Organism in India. (Ramanujam, B. and Rabindra, R.J. eds.). Bangalore, India. PDBC Publications, pp124-134.

Bai, Y.; Zhou-Xiao, M. and Smith, D.L. 2003. Enhanced soybean plant growth resulting from co-inoculation of Bacillus strains with Bradyrhizobium japonicum. Crop Sci., 43: 1774-1781.

Bakker, P.A.; Pieterse, C.M. and Van Loon, L.C. 2007. Induced Systemic Resistance by Fluorescent Pseudomonas spp. Phytopathology, 97: 239-43.

Bapiri, A.; Asgharzadesh, A.; Mujallali, H.; Khavazi, K. and Pazira, E. 2012. Evaluation of zinc solubilization potential by different strains of fluorescent pseudomonads. J. Appl. Sci. Environ. Manage., 16: 295-298.

Barea, J.M.; Pozo, M.J.; Azcón, R. and Azcón-Aguilar, C. 2005. Microbial cooperation in the rhizosphere. J. Exp. Bot., 56: 1761-1778.

Beatty, P.H. and Jensen, S.E. 2002. Paenibacillus polymyxa produces fusaricidin type antifungal antibiotics active against Leptosphaeria maculans, the causative agent of blackleg disease of canola. Can. J. Microbiol., 48: 159-169.

Borowicz, V. 2001. Do arbuscular mycorrhizal fungi alter plant pathogen relations? Ecology, 82: 3057-3068.

Bradley, C.A.; Hartman, G.L.; Nelson, R.L.; Mueller, D.S. and Pedersen, W.L. 2001. Response of ancestral soybean lines and commercial cultivars to Rhizoctonia root and hypocotyl rot. Plant Dis., 85: 1091-1095.

Budi, S.W.; Van Tuinen, D.; Martinotti, G. and Gianinazzi, S. 1999. Isolation from the Sorghum bicolor mycorrhizosphere of a bacterium compatible with arbuscular mycorrhiza development and antagonistic towards soilborne fungal pathogens. Appl. Environ. Microbiol., 65: 5148-5150. 
Carvalho, F.P. 2017. Pesticides, environment, and food safety. Rev. Food and Ener. Secur., 6 (2): 48-60.

Chakraborty, M.R. and Chatterjee, N.C. 2007. Interaction of Trichoderma harzianum with Fusarium solani during its pathogenesis and the associated resistance of the host. Asian J. Exp. Sci., 21: 351-355.

Chang, K.F.; Hwang S.F.; Ahmed, H.U.; Strelkov, S.E.; Turnbull, G.D.; Burke, D. A. and Harding, M.W. 2015. Response of soybean cultivars and lines to seedling blight and root rot (Rhizoctonia solani) under field conditions in southern Alberta. Can. J. Plant Pathol., 38: 262. [Abstract].

Choudhary, D.K.; Varma, A. and Tuteja, N. 2017. Mycorrhizal Helper Bacteria: Sustainable Approach. In: Mycorrhiza - Function, Diversity, State of the Art. (Varma, A.; Prasad R. and Tuteja, N. eds.). Springer International Publishing, Cham, Switzerland, pp 61-74.

Cottenie, A.; Verloo, M.; Kiekns, L.; Velghe, G. and Camerlynek, R. 1982. Chemical Analysis of Plants and Soils. Laboratory of Analytical and Agrochemistry. State University, Ghent Belgium. 63p.

Da Mota, F.F.; Gomes, E.A. and Seldin, L. 2008. Auxin production and detection of the gene coding for the auxin efflux carrier (AEC) protein in Paenibacillus polymyxa. J. Microbiol., 56: 275-264.

Dwivedi, D.; Johri, B.N.; Ineichen, K.; Wray, V. and Wiemken, A. 2009. Impact of antifungals producing rhizobacteria on the performance of Vigna radiata in the presence of arbuscular mycorrhizal fungi. Mycorrhiza, 19: 559-570.

Farkas, G.L. and Kiraly, Z. 1962. Role of phenolic compounds in the physiology of plant diseases and disease resistance. Phytopath., Z., 44: 105-150.

Fukui, R.; Schroth, M.N.; Hendson, M. and Hancock, J.G. 1994. Interaction between strains of Pseudomonads in sugar beet spermospheres and the relationship to pericarp colonization by Pythium ultimum in soil. Phytopathology, 84: 13221330.

Gamalero, E.; Martinotti, M.G.; Trotta, A.; Lemanceau, P. and Berta, G. 2004. Morphogenetic modifications induced by Pseudomonas fluorescens A6RI and Glomus mosseae BEG12 in the root system of tomato differ according to plant growth conditions. New Phytol., 155: 293-300.

Garbaye, J. 1994. Helper bacteria: a new dimension to the mycorrhizal symbiosis. New Phytologist, 128: 197-210.

Goodman, R.N.; Kiraly, Z. and Zaitlin, M. 1967. The Biochemistry and Physiology of Infectious plant Diseases. Van Nostrand Co. Inc., Prince-ton, New Jersey. $354 \mathrm{p}$.

Guetsky, R.; Shtienberg, D.; Elad, Y.; Fischer, E. and Dinoor, A. 2002. Improving biological control by combining biocontrol agents each with several mechanisms of disease suppression. Phytopathology, 92: 976-985. 
Gupta, A. and Gopal, M. 2008. Siderophore production by plant growth promoting rhizobacteria. Indian J. Agric. Res., 42 (2): 153 -156.

He, Z.; Kisla, D.; Zhang, L.; Yuan, C.H.; Green-Church, K.B. and Yousef, A.E. 2007. Isolation and identification of a Paenibacillus polymyxa strain that coproduces a novel lantibiotic and polymyxin. Appl. Environ. Microbiol., 73: $168-178$.

Islam, M.T.; Deora, A.; Hashidokoa, Y.; Rahmana, A.; Itoa, T. and Tahara, S. 2007. Isolation and identification of potential phosphate solubilizing bacteria from the rhizoplane of Oryza sativa L. cv. BR29 of Bangladesh. Z. Naturforsch, 62(c): 103-110.

Jäderlund, L.; Arthurson, V.; Granhall, U. and Jansson, J.K. 2008. Specific interactions between arbuscular mycorrhizal fungi and plant growth promoting bacteria: as revealed by different combinations. FEMS Microbiol. Lett., 287: 174-180.

Koske, R.E. and Gemma, J.N. 1989. A modified procedure for staining roots to detect VA mycorrhizas. Mycol. Res., 92: 486-488.

Larkin, R.P.; Roberts, D.P. and Gracia-Garza, J. A. 1998. Biological control of fungal diseases. In: Fungicidal Activity-Chemical and Biological Approaches to Plant Protection. (Hutson, D. and Miyamoto, J. eds.). Wiley, New York, NY., pp 141-191.

Linderman, R.G. 1994. Role of VAM fungi in biocontrol. In: Mycorrhizae and plant health. (Bethlenfalvay, G. J. and Linderman R. G. eds.). APS Press, St Paul, Minnesota, pp 1-26.

Linderman, R.G. 1997. Vesicular arbuscular mycorrhizal (VAM) fungi. In: The Mycota, vol. V, Plant Relationships Part B. (Carroll, G. C., and Tudzynski, P. eds.). Berlin, Germany, Springer-Verlag, pp 117-128.

Lisette, J.; Xavier, C. and Germida, J.J. 2003. Selective interactions between arbuscular mycorrhizal fungi and Rhizobium leguminosarum bv. viceae enhance pea yield and nutrition. Biol. Fertil. Soils, 37: 261-267.

Michelsen, C.F.; Watrous, J.; Glaring, M.A.; Kersten, R.; Koyama, N. and Dorrestein, P.C. 2015. Nonribosomal peptides, key biocontrol components for Pseudomonas fluorescens In5, isolated from a Greenlandic suppressive soil. mBio, 6 (2) e00079-15.

Mortimer, P.E.; Pe'rez-Ferna'ndez, M.A. and Valentine, A.J. 2009. Arbuscular mycorrhizae affect the $\mathrm{N}$ and $\mathrm{C}$ economy of nodulated Phaseolus vulgaris (L.) during $\mathrm{NH}_{4}{ }^{+}$nutrition. Soil Biol. Biochem., 41: 2115-2121.

Nicholson, R.L. and Hammerschmidt, R. 1992. Phenolic compounds and their role in disease resistance. Ann. Rev. Phytopathol., 30: 369-389.

Nishijima, F.; Evans, W.R. and Vesper, S.J. 1988. Enhanced nodulation of soybean by Bradyrhizobium in the presence of Pseudomonas fluorescens. Plant Soil, 111: $149-150$. 
O'Callaghan, M. 2016. Microbial inoculation of seed for improved crop performance: issues and opportunities. Appl. Microbiol. Biotechnol., 100: 5729-5746.

Page, A.L.; Miler, R.H. and Keeney, D.R. 1982. "Methods of Soil Analysis", part 2. Chemical and microbiological properties. Agronomy monographs No. 9, $2^{\text {nd }}$ ed., pp. 539-624.

Pérez-de-Luque, A.; Tille, S.; Johnson, I.; Pascual-Pardo, D.; Ton, J. and Cameron, D.D. 2017. The interactive effects of arbuscular mycorrhiza and plant growth promoting rhizobacteria synergistically enhance host plant defences against pathogens. Scientific Reports, 7:16409.

Pertot, I.; Giovannini, O.; Benanchi, M.; Caffi, T.; Rossi, V. and Mugnai, L. 2017. Combining biocontrol agents with different mechanisms of action in a strategy to control Botrytis cinerea on grapevine. Crop Protec., 97: 85-93.

Priyadharsini, P. and Muthukumar, T. 2016. Interactions between Arbuscular Mycorrhizal Fungi and potassium solubilizing microorganisms on agricultural productivity. In: Potassium Solubilizing Microorganisms for Sustainable Agriculture. (Meena, V.; Maurya, B.; Verma, J. and Meena, R. eds.). Springer, New Delhi, pp 111-125.

Priyadharsini, P.; Rojamala, K.; Ravi, R.K.; Muthuraja, R.; Nagaraj, K. and Muthukumar, T. 2016. Mycorrhizosphere: The Extended Rhizosphere and Its Significance.. In: Plant-Microbe Interaction: An Approach to Sustainable Agriculture. (Choudhary, D.; Varma, A. and Tuteja, N. eds.). Springer, Singapore. Pp 97-124.

Raza, W. and Shen, Q. 2010. Growth, $\mathrm{Fe}_{3}{ }^{+}$reductase activity, and siderophore production by Paenibacillus polymyxa SQR-21 under differential iron conditions. Curr. Microbiol., 61: 390-395.

Roberts, D.P.; Lohrke, S.M.; Meyer, S.L.F.; Buyer, J.S.; Bowers, J.H.; Baker, C.J.; Li, W.; de Souza, J.T.; Lewis, J.A. and Chung, S. 2005. Biocontrol agents applied individually and in combination for suppression of soil-borne diseases of cucumber. Crop Protec., 24: 141-155.

Rouphael, Y.; Franken, P.; Schneider, C.; Schwarz, D.; Giovannetti, M. and Agnolucci, M. 2015. Arbuscular mycorrhizal fungi act as bio-stimulants in horticultural crops. Sci. Hort., 196: 91-108.

Sadasivam, S. and Manickam, A. 1996. Biochemical Methods. $2^{\text {nd }}$ ed. New Age Int. Pvt. Ltd. Pub. \& T.N. Agric. Univ. Coimbatore, Tamil Nadu, India. pp. 108110.

Sampangi, R.K. and Bagyaraj, D.J. 1989. Root Diseases and Mycorrhizae. J. Phytopathol. Res., 2: 1-6.

Sayeed, A.M.S. and Siddiqui, Z.A. 2008. Biocontrol of a root rot disease complex of chickpea by Glomus intraradices, Rhizobium sp. and Pseudomonas straita. Crop Protec., 27: 410-417. 
Sharma, Y.R. 2006. Recent Trends in the Use of Antagonistic Organism for Disease Management in Spice Crops. In: Current Status of Biological Control of Plant Diseases Using Antagonistic Organism in India. (Ramanujam, B. and Rabindra, R.J. eds.). Bangalore, India: PDBC publications, pp 49-73.

Shehata, S.T.; Mosbah, M.M. and Hegazi, M.F. 2006. Selection of biocontrol agents for control of onion white rot disease. J. Agric. Sci. Mansoura Univ., 31: 26212637.

Silva, F. and Azevedo, C.A.V. 2009. Principal components analysis in the software Assistat-Statistical Attendance. In: World Congress on Computers in Agriculture, 7. Orlando Proceeding, American Society of Agricultural and Biological Engineers.

Silva, V.N.; Silva, L.E.S.F.; Martinez, C.R.; Seldin, L.; Burity, H.A. and Figueiredo, M.V.B. 2007. Effect of Paenibacillus strains on specific nodulation in Bradyrhizobium-Bacillus symbiosis. Acta Sci. Agron., 29: 331-338.

Singh. R.; Soni. S.K. and Kalra, A. 2012. Synergy between Glomus fasciculatum and a beneficial Pseudomonas in reducing root diseases and improving yield and forskolin content in Coleus forskohlii Briq under organic field conditions. Mycorrhiza, 23(1): 35-44.

Snell, F.D. and Snell, C.T. 1953. Calorimetric methods of analysis, including some turbidimetric and nephelometric methods. $3^{\text {rd }}$ ed., Volume III (Organic I), D. Van Nostrand CO. Inc., Princeton, NJ, USA. 606p.

Solanki, M.K.; Singh, R.K.; Srivastava, S.; Kumar, S.; Kashyap, P.L.; Srivastava, A. K. and Arora, D.K. 2014. Isolation and characterization of siderophore producing antagonistic rhizobacteria against Rhizoctonia solani. J. Basic Microbiol., 54: 585-596.

Souchie, E.L.; Azcón, R.; Barea, J.M.; Saggin-Júnior, O.J. and Ribeiro da Silva, E. M. 2003. Indole acetic acid production by P-solubilizing microorganisms and interaction with arbuscular mycorrhizal fungi. Acta Sci. Biol. Sci., 29: 315-320.

St-Arnaud, M. and Elsen, A. 2005. Interaction of arbuscular mycorrhizal fungi with soil-borne pathogens and non-pathogenic rhizosphere micro-organisms. In: In vitro culture of mycorrhizas. (Declerck, S.; Strullu, S. G.; Fortin J.A. eds.). Springer-Verlag, Berlin Heidelberg, pp 217-231.

Susilowati, A.; Wahyudi, A.T.; Lestari, Y.; Suwanto, A. and Wiyono, S. 2011. Potential Pseudomonas isolated from soybean rhizosphere as a biocontrol against soilborne phytopathogenic fungi. HAYATI Journal of Biosciences, 18 (2): 51-56.

Sutha, R.; Ramiah, M. and Rajappan, K. 1998. Changes in protein and amino acid composition of tomato due to a tospovirus infection. Indian Phytopath., 51: 136-139. 
Taranto, F.; Pasqualone, A.; Mangini, G.; Tripodi, P.; Miazzi, M.M.; Pavan, S. and Montemurro, C. 2017. Polyphenol oxidases in crops: biochemical, physiological and genetic aspects. Int. J. Mol. Sci. 18, 377.

Timmusk, S.; Nicander, B.; Granhall, U. and Tillberg, E. 1999. Cytokinin production by Paenibacillus polymyxa. Soil Biol. Biochem., 31: 1847-1852.

Toussaint, J.P.; Kraml, M.; Nell, M.; Smith, S.E.; Smith, F.A.; Steinkellner, S.; Schmiderer, C.; Vierheilig, H. and Novak J. 2008. Effect of Glomus mosseae on concentrations of rosmarinic and caffeic acids and essential oil compounds in basil inoculated with Fusarium oxysporum f.sp. basilici. Plant Pathol., 57: 1109-1116.

Toussaint, J.P.; ST-Arnaud, M. and Charest, C. 2004. Nitrogen transfer and assimilation between the arbuscular mycorrhizal fungus Glomus intraradices Schench and Smith and Ri T-DNA roots of Daucus carota L. an in vitro compartment system. Can. J. Microbiol., 50: 251- 260.

Tripathi, S.; Mishra, S.K. and Varma, A. 2017. Mycorrhizal Fungi as Control Agents Against Plant Pathogens. In: Mycorrhiza - Nutrient Uptake, Biocontrol, Ecorestoration. (Varma, A.; Prasad, R. and Tuteja, N. eds.). Fourth Edition, Springer International Publishing, Switzerland, pp 161-178.

Van Loon, L.C.; Rep, M. and Pieterse, C.M.J. 2006. Significance of inducible defence related proteins in infected plants. Ann. Rev. Phytopath., 44: 135-162.

Wang, F.; Gao, R.J.; Li, J.O.; Wu, X.H. and Geny, P.T. 2000. Effect of difenoconazole on the growth and activities of disease resistance related enzymes in wheat seedlings from treated seeds. Acta Phytopathol. Sinica, 30: 213-216.

Xavier, L.J.C. and Germida, J.J. 2003. Bacteria associated with Glomus clarum spores influence mycorrhizal activity. Soil Biol. Biochem., 35: 471-478.

Xue, A.G.; Cober, E.; Morrison, M.J.; Voldeng, H.D. and Ma, B.L. 2007. Effect of seed treatments on emergence, yield, and root rot severity of soybean under Rhizoctonia solani inoculated field conditions in Ontario. Can. J. Plant Sci., 87: $167-173$.

Yao, M.K.; Tweddell, R.J. and Desilets, H. 2002. Effect of two vesicular-arbuscular mycorrhizal fungi on the growth of micro-propagated potato plantlets and on the extent of diseases caused by Rhizoctonia solani. Mycorrhiza, 12: 235-242.

Yoruk, R. and Marshall, M.R. 2003. Physicochemical properties and function of plant polyphenol oxidase: a review. J. Food Biochem., 27: 361-422.

Zhang, Q.; Ji, Y.; Xiao, Q.; Chng, S.; Tong, Y.; Chen, X. and Liu, F. 2016. Role of Vfr in the regulation of antifungal compound production by Pseudomonas fluorescens FD6. Microbiol. Res., 188-189: 106-112.

(Received 03/06/2018; in revised form 08/07/2018) 


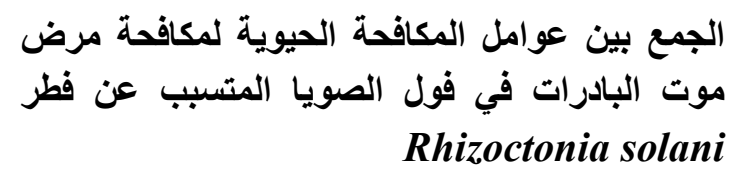

\author{
مروى عبد الله محمود عطوه \\ قسم بحوث أمر اض الهحاصيل البقولية و العلف \\ معهد بحوث أمر اض النباتات ـ مركز البحوث الزبل الزر اعبة ـ الجيزة
}

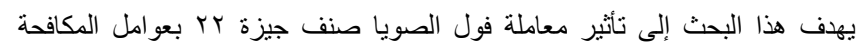

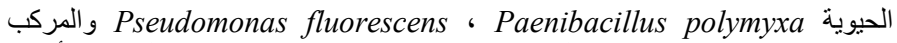
الحيوي ميكوريزين (يحتوي بشكل رئيسي علي الجنس Glomus) إما بشكل فردي أو في

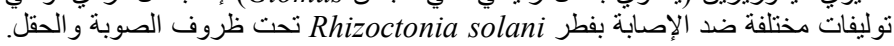

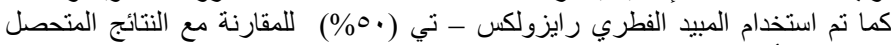

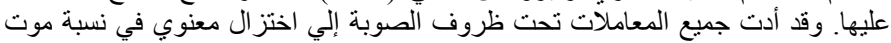

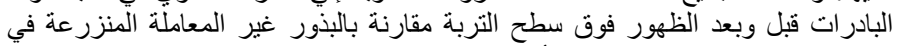

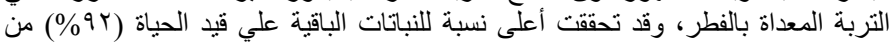

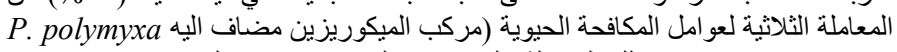

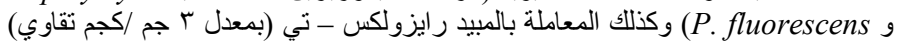

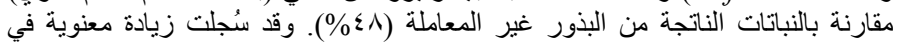

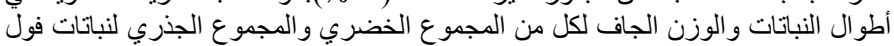

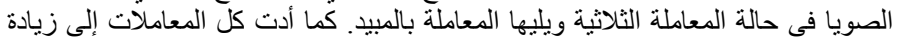

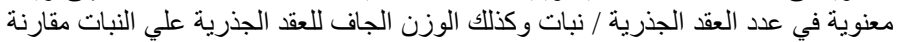

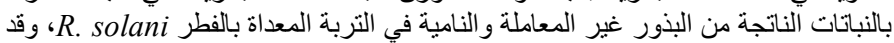

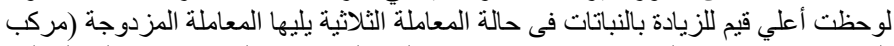

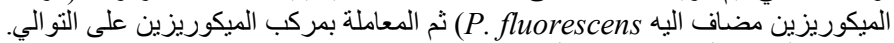

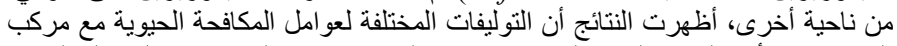

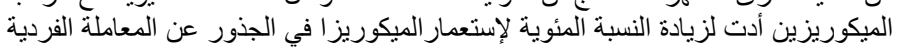

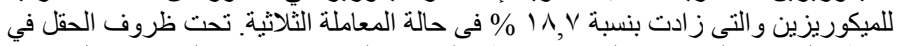

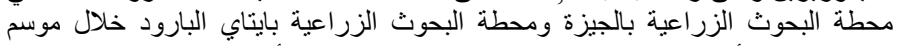

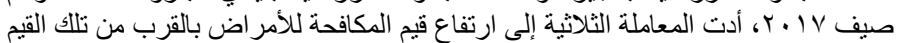

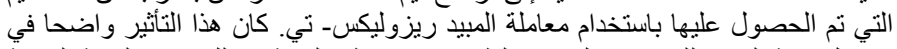

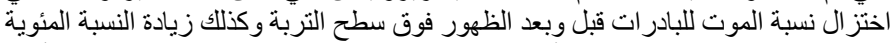

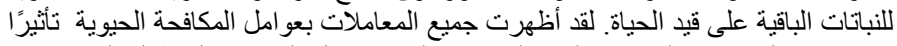

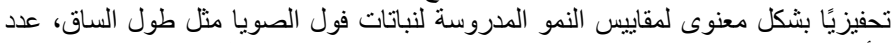

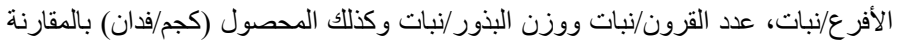

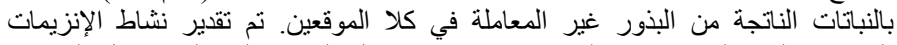

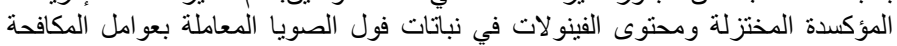

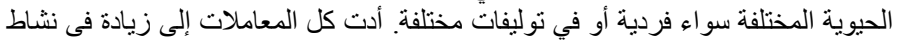

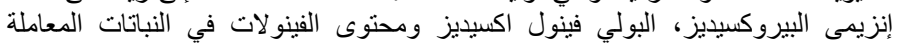

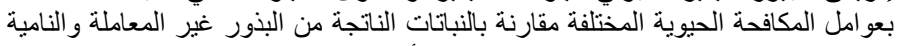

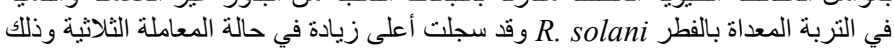

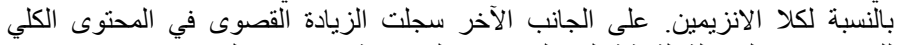
للفينو لات في المعاملة الثلاثية يليها المعاملات المزدوجة مع مركب الميكوريزين. 